\title{
The 2021 Metabolomics publication awards
}

\section{Royston Goodacre ${ }^{1}$}

๑) Springer Science+Business Media, LLC, part of Springer Nature 2021
Every year Metabolomics publishes some fantastic papers and some of these we are delighted to say are extremely well received by the community. Several years ago we decide that it would be nice to highlight these excellent papers and reviews and thus Metabolomics awards the following prizes to the most downloaded original articles and reviews published in the previous year:

- Best Paper Award - this is awarded to the Primary Research Article with the highest total number of downloads during the previous year. The first author of the winning paper receives a complimentary ebook from Springer (value 200€), and we highlight the work on the front cover of our Metabolomics journal. The paper will be made freely available for 30 days.

- The runner-up paper will also be made freely available for 30 days.

- Best Review Award - this is awarded to the Review Article with the highest total number of downloads during the previous year. The first author of the winning paper receives complimentary e-books from Springer (value $200 €$ ), we also highlight the review on the front cover of our Metabolomics journal. The review will also be made freely available for 30 days.

- The runner-up review will also be made freely available for 30 days.

For a paper to be eligible for these awards it must be either a Primary Research Paper (which may include best practice or standards articles, as well as letters and short communications) or a Review Article (which includes white papers) that was published in its final form in 2020. As mentioned above all awards will be based on their popularity via downloads.

Royston Goodacre

roy.goodacre@liverpool.ac.uk

1 Department of Biochemistry and Systems Biology, Institute of Systems, Molecular and Integrative Biology, University of Liverpool, Biosciences Building, Crown Street, Liverpool L69 7ZB, UK
Congratulations to the following 2021 winners!!!

\section{Best Paper Award published in 2020}

Winner-

- Mendez, M. M., Broadhurst, D. I., \& Reinke, S. N. (2020). Migrating from partial least squares discriminant analysis to artificial neural networks: a comparison of functionally equivalent visualisation and feature contribution tools using Jupyter notebooks. Metabolomics 16: 17. https://doi.org/10.1007/s11306-020-1640-0

Runner up-

- Abuawad, A., Mbadugha, C., Ghaemmaghami, A. M.. \& Kim, D.-H. (2020). Metabolic characterisation of THP-1 macrophage polarisation using LC-MS-based metabolite profiling. Metabolomics 16: 33. https://doi.org/10.1007/ s11306-020-01656-4

\section{Best Review Award published in 2020}

Winner-

- O'Shea, K., \& Misra, B. B. (2020). Software tools, databases and resources in metabolomics: Updates from 2018 to 2019. Metabolomics, 16: 36. https://doi.org/10.1007/ s11306-020-01657-3

\section{Runner up-}

- Bedair, M., \& Glenn, K. C. (2020). Evaluation of the use of untargeted metabolomics in the safety assessment of genetically modified crops. Metabolomics, 16: 111. https://doi.org/10.1007/s11306-020-01733-8

Publisher's Note Springer Nature remains neutral with regard to jurisdictional claims in published maps and institutional affiliations. 